\title{
Plasmid DNA delivery into MDA-MB-453 cells mediated by recombinant Her-NLS fusion protein
}

This article was published in the following Dove Press journal:

International Journal of Nanomedicine

18 September 2010

Number of times this article has been viewed

\section{Sivakumar Jeyarajan* \\ Jennifer Xavier* \\ N Madhusudhana Rao \\ Vijaya Gopal}

Centre for Cellular and Molecular Biology, Council for Scientific and Industrial Research, Hyderabad, Andhra Pradesh, India; *These authors contributed equally to this work
Correspondence: Vijaya Gopal Centre for Cellular and Molecular Biology, Council for Scientific and Industrial Research, Uppal Road, Hyderabad 500007, Andhra Pradesh, India

$\mathrm{Tel}+9 \mid 4027192552$

$\mathrm{Fax}+91402716059 \mid$

Email vijaya@ccmb.res.in
Abstract: A major rate-limiting step in nonviral gene delivery is the entry of nucleic acids across various membrane barriers and eventually into the nucleus where it must be transcribed. Cell-penetrating peptides and proteins are employed to generate formulations that overcome these challenges to facilitate DNA delivery into cells efficiently. However, these are limited by their inability to deliver nucleic acids selectively due to lack of specificity because they deliver to both cancer and normal cells. In this study, through modular design, we generated a recombinant fusion protein designated as Her-nuclear localization sequence (Her-NLS), where heregulin- $\alpha$ (Her), a targeting moiety, was cloned in frame with cationic NLS peptide to obtain a cell-specific targeting biomolecule for nucleic acid delivery. The heregulin- $\alpha_{1}$ isoform possesses the epidermal growth factor-like domain and binds to HER $2 / 3$ heterodimers which are overexpressed in certain breast cancers. Purified recombinant Her-NLS fusion protein binds plasmid DNA and specifically transfects MDA-MB-453 cells overexpressing the epidermal growth factor receptors HER2/3 in vitro. The approach described would also permit replacement of heregulin ligand with other targeting moieties that would be suited to cell-specific nucleic acid delivery mediated via receptor-ligand interactions.

Keywords: cell targeting, nucleic acid delivery, nuclear localization sequences, heregulin, transfection, HER2 receptors

\section{Introduction}

The design of vehicles for safe and efficient delivery of nucleic acids into cells is critical to successful gene therapy protocols. However, the major obstacle is lack of efficient delivery methodologies. Peptide-based delivery systems permit generation of diverse molecules that can potentially transfect and target cells with minimal toxicity, and often efficiency is linked to design. Cell-penetrating peptides (CPPs) in gene delivery protocols, in the last decade, have gained attention due to their versatility, ${ }^{1-3}$ because they are rich in basic residues, such as lysine and arginine. These peptides are able to condense DNA efficiently into small, compact nanoparticles through electrostatic interactions to generate multifunctional formulations of therapeutic value. The advantages derived from short peptides in gene delivery protocols are due to their potential to overcome various barriers, including cell, endosomal, and nuclear membrane barriers. We recently characterized and explored the utility of several designed recombinant peptides that are cationic in nature, nontoxic, and have novel transfection properties. ${ }^{4,5}$ These multidomain fusion peptides are primarily utilized in gene therapy protocols due to their increased efficiency that is comparable with that of existing commercial formulations. 
CPPs lack sequence similarity and specificity. However, due to their cationic nature, cell-penetrating peptides have been used successfully to bind DNA and transfect cells. One of the early examples proposed for cell-specific DNA transfer is based on the chimeric multidomain fusion protein, where a single chain antibody domain that binds to the ErbB-2 receptor was cloned with the exotoxin translocation domain of Pseudomonas and a DNA-binding domain. ${ }^{6}$ Complexes of plasmid DNA with the multidomain fusion protein were able to transfect ErbB-2-expressing tumor cells in vitro in a cellspecific manner. ErbB-2, the human epidermal growth factor receptor, HER2/neu is a transmembrane protein that belongs to the epidermal growth factor receptor family. This gene is overexpressed in $15 \%-20 \%$ of breast cancers. Therapeutic agents against HER2/neu have been intensively sought over the last few years ${ }^{7,8}$ to treat a multitude of cancers. Recently, a study described the use of CPPs such as HIV-TAT conjugated to peptide mimetic carriers, to target ErbB2-overexpressing breast cancers specifically. Being a transducing moiety, TAT makes internalization highly efficient while simultaneously delivering conjugated molecules. ${ }^{9}$ Bidewell et al have investigated the ability to target specific compartments ${ }^{10}$ with CPPs that are beneficial in cancer therapy. In addition, the TAT epitope possesses an inherent nuclear localization signal that contributes to the enhanced transfection efficiencies observed by several laboratories. It is thus evident that the conjugation of peptide ligands to CPPs will permit targeting to receptors in a cell-specific manner and are therefore natural and safer alternatives to viral vectors.

Heregulin- $\alpha$ was originally purified from conditioned medium $^{11}$ in a human breast cancer cell line, and identified to represent the natural ligand for HER2/ErbB2 with a single high affinity binding site. HER2/neu protein overexpression has been observed in non-small cell lung cancer, especially in lung adenocarcinoma. ${ }^{12}$ HER2 receptors are involved in the pathogenesis of various types of malignancies, including prostate, breast, ovarian, and lung cancer, and are therefore a good target for the specific introduction of nucleic acids into the cell. Through these studies, ErbB2 has been shown to be an ideal target for cancer therapies. Nearly a decade ago, a chimeric fusion of 10 lysine residues to the penton base of the adenoviral capsid protein was cloned in frame with the epidermal growth factor-like domain of the heregulin- $\alpha 1$ isoform that targets breast cancer cells in a cell-specific manner. ${ }^{13}$ Such an approach may reduce the amount that is required for targeting with reduced toxicity. Although there have been considerable efforts worldwide to design efficient targeting vectors, the limitations of current DNA transfection protocols have driven our attempt to identify and create suitable transfection-efficient biomolecules for cell-specific targeting. Designer peptides with distinct DNA-binding epitopes or nuclear localization epitopes serve as nucleic acid binders, while the ligand that recognizes its corresponding receptor will render cell specificity, thereby facilitating internalization of nucleic acids into cells.

Cell targeting has been demonstrated in yet another effort where a multidomain fusion protein was engineered to target cancer cells and also destabilize endosomal membranes once internalized, ${ }^{14}$ thereby mediating efficient DNA delivery. Strategies for delivering plasmids are also being used to deliver other therapeutic nucleic acids, such as short interfering RNA (siRNA). ${ }^{7} 15$ Recombinant cationic fusion proteins can thus be harnessed to carry and deliver all types of nucleic acids in a cell-specific manner.

We recently demonstrated that fusion peptide TATNLS-Mu (TNM), with three nuclear localization sequence (NLS) epitopes was more efficient than TAT-Mu $\mathrm{Mu}^{4}$ which is devoid of these epitopes, suggesting a role of NLS in the engineered recombinant TNM peptide. In an elegant study, Subramanian et al conjugated the NLS motifs for DNA delivery by conjugating a nonclassical NLS M9 peptide $^{16}$ that resulted in increased reporter gene expression. Here, we report the generation of a heterologous His-tag fusion protein where a plasmid construct with three NLS moieties in tandem were cloned in frame with the heregulin- $\alpha$ moiety, resulting in a chimera designated as Her-NLS, with the premise that the NLS epitope would facilitate the passage of DNA into the nucleus once delivered into the cytoplasm. The cloned heregulin- $\alpha$ moiety, with an epidermal growth factor-like domain would enable efficient targeting of DNA via specific receptors overexpressed in breast carcinoma cell lines. The ability to bind DNA, cell uptake, and the cell biologic activity of purified recombinant protein that harbors the heregulin ligand fused in-frame with the NLS epitopes was investigated and demonstrated in this study.

\section{Methods \\ Cells, plasmid constructs, and fusion peptides}

Plasmids pHerPBK10, pRSET-Her, MDA-MB-231, and MDA-MB-453 human breast cancer cells was obtained from Medina-Lali Kauwe, University of Southern California Keck School of Medicine, Los Angeles, CA. Plasmids pEG$\mathrm{FPN}_{3}$ and pCMV $\beta$-gal were from inhouse stocks. Plasmid pEYFPNuc was obtained from David Dean's laboratory, presently at the University of Rochester (Rochester, NY). 
These cells were grown and maintained in Dulbecco's modified Eagle's medium (DMEM)-F12, 10\% fetal calf serum at $37^{\circ} \mathrm{C}$, and $5 \% \mathrm{CO}_{2}$. Oregon Green nucleic acid labeling kit was from Invitrogen (Eugene, OR). Protamine sulfate was from Sigma-Aldrich (St. Louis, MO). All other reagents were of the highest purity.

\section{Construction and production of recombinant protein Her-NLS in Escherichia coli}

Plasmid pTAT-NLS was generated by amplifying the NLS moiety from $p E Y F P N u c$ and cloning in frame with the TAT epitope of pTAT. For generating cell specificity and cloning the cell-targeting ligand construct, the TAT moiety was deleted and the sites were used for inserting the polymerase chain reaction-amplified heregulin moiety. The gene for heregulin, the targeting ligand $(0.6 \mathrm{~kb})$, was amplified by polymerase chain reaction (PCR) from the plasmid pHerPBK10, using forward primer- 5'TCTGTACGACGATGACGATAAG-3', and reverse primer-5'-GCATGGATCCACTTCCAC-3' with BamHI sites incorporated for generating pHer-NLS. These plasmid constructs were verified by DNA sequencing to confirm the integrity of the cloned heregulin- $\alpha$ targeting ligand and the DNA binding epitope. pHer-NLS was then transformed into Escherichia coli expression host BL21(DE3)pLysS and induced with isopropyl-beta-Dthiogalactopyranoside (IPTG). The histidine tag of the fusion protein Her-NLS facilitates purification with cobalt affinity matrix. The predicted molecular mass is 34,699 Daltons.

\section{Overexpression and purification of recombinant proteins under native conditions}

Plasmid pHer-NLS was transformed into BL21(DE3)pLysScompetent cells plated on Luria Bertani plates containing $34 \mu \mathrm{g} / \mathrm{mL}$ of chloramphenicol and $100 \mu \mathrm{g} / \mathrm{mL}$ of ampicillin. Cultures were grown at $30^{\circ} \mathrm{C}$ until mid-log phase. Following induction with $1 \mathrm{mM}$ IPTG, cells were grown at $20^{\circ} \mathrm{C}$ for eight hours. Briefly, bacterial cultures were harvested and the pellet was suspended in $25 \mathrm{~mL}$ lysis buffer $(50 \mathrm{mM}$ sodium phosphate buffer $\mathrm{pH} 7.0,500 \mathrm{mM} \mathrm{NaCl}, 1 \mathrm{mM}$ phenylmethylsulfonyl fluoride, and a cocktail protease inhibitor tablet) for 15 minutes and sonicated. The lysate was centrifuged at $10,000 \mathrm{rpm}$ for 30 minutes and filtered using $0.45 \mu \mathrm{m}$ filters; $1 \mathrm{~mL}$ of cobalt resin was added to the filtered supernatant, pre-equilibrated with lysis buffer, and incubated at $4^{\circ} \mathrm{C}$ for one hour. The resin containing the bound protein was washed with $10 \mathrm{~mL}$ wash buffer $(50 \mathrm{mM}$ sodium phosphate buffer $\mathrm{pH}$ 7.0, $500 \mathrm{mM} \mathrm{NaCl}, 10 \mathrm{mM}$ imidazole, $1 \mathrm{mM}$ phenylmethylsulfonyl fluoride and eluted with $0.5 \mathrm{~mL}$ elution buffer (50 mM sodium phosphate buffer $\mathrm{pH} 7.0,500 \mathrm{mM} \mathrm{NaCl}$, $250 \mathrm{mM}$ imidazole, $1 \mathrm{mM}$ phenylmethylsulfonyl fluoride, and a cocktail protease inhibitor tablet) in four fractions. The use of a cobalt affinity matrix (BD TALON; BD Biosciences, Palo Alto, CA) eliminated the binding of endogenous contaminating E. coli histidine-rich protein. The fractions were analyzed on $12 \%$ sodium dodecyl sulfate polyacrylamide gel electrophoresis (SDS-PAGE), pooled, and passed through PD-10 desalting columns (BD Biosciences) with phosphatebuffered saline at $\mathrm{pH}$ 7.4. The corresponding molecular mass of protein Her-NLS was analyzed by matrix-assisted laser desorption/ionization time of flight mass spectrometry (MALDI-TOFMS). The final yield was typically $2 \mathrm{mg}$ from a $1000 \mathrm{~mL}$ culture where the protein was greater than $95 \%$ homogeneous, as ascertained by SDS-PAGE.

\section{Western blot analysis}

Immunostaining of Her-NLS with anti-His antibody Western blotting was done with purified proteins using monoclonal anti-His-antibodies against the $6 \times$ His-tag fusion proteins, followed by binding to the secondary antibody coupled to alkaline phosphatase as described. ${ }^{5}$

\section{HER2 receptor expression in breast cancer cells by immunostaining}

Cell lysates for receptor quantitation prepared in $250 \mathrm{mM}$ Tris- $\mathrm{Cl} \mathrm{pH}$ 8.0, 0.5\% NP-40 lysis buffer, and equal protein were loaded on a $6 \%$ sodium dodecyl sulfate gel after sonication. After electrophoresis, proteins were electroblotted onto a nitrocellulose membrane. Blots were probed with monoclonal antibody, anti-ErbB2 (intracellular domain) at 1:200 dilution and incubated overnight at $4^{\circ} \mathrm{C}$, followed by incubation with a secondary antibody, antimouse-ALP (Bangalore Genei, Bangalore, India) 1:4000 for one hour at room temperature. Reactive bands were visualized with nitro blue tetrazolium chloride and 5-bromo-4-chloro-3-indoyl-1-phosphate.

\section{DNA binding, electrophoretic mobility shift, and protection assays}

Charge neutralization and DNA condensation of the fusion protein were observed by electrophoresis of the complexes on agarose gels as described previously. ${ }^{5}$ Briefly, plasmid DNA pEGFPN ${ }_{3}(0.2 \mu \mathrm{g})$ was complexed with increasing amounts of protein Her-NLS to obtain the corresponding charge ratios, in Hepes-buffered saline $(150 \mathrm{mM} \mathrm{NaCl}, 5 \mathrm{mM}$ 
$\mathrm{KCl}, 0.75 \mathrm{mM} \mathrm{Na}_{2} \mathrm{HPO}_{4}$, and $20 \mathrm{mM}$ Hepes (pH 7.4) and incubated for 25 minutes at room temperature. The charge ratio of plasmid DNA to the fusion protein Her-NLS is indicated as D:P, and is calculated based on the contribution by the negatively charged phosphates on DNA that was kept constant. The number of positive charges originating from Her-NLS is 51 and protamine sulfate is 20. In the case of DNA:Her-NLS:protamine sulfate, formulated at a 1:8:2 charge ratio, protamine sulfate was added at $2 \mathrm{nmol}$ of positive charge per nmol DNA and 8 nmol of Her-NLS. Complexes were then treated with DNase I at the indicated concentrations and processed by extraction with a mixture of phenol-chloroform. The complexes were subsequently analyzed on $1 \%$ agarose gel (Tris-acetate buffer system, $\mathrm{pH}$ 8.2) and stained with ethidium bromide post electrophoresis. Binding is observed as retardation in the migration of the D:P complexes on an agarose gel.

\section{Live uptake of plasmid pEGFPN mediated by Her-NLS using confocal microscopy}

MDA-MB-453 and MDA-MB- 231 cells were plated to about $70 \%$ confluence on chambered coverglass (LabTek, Nalge Nunc International Corp., Rochester, NY) and washed with serum-free DMEM ( $\mathrm{pH} 7.4)$. Plasmid DNA pEGFPN $_{3}(0.45 \mu \mathrm{g})$ labeled with Oregon Green (Ex $\lambda$ $493 \mathrm{~nm}, \operatorname{Em} \lambda 520 \mathrm{~nm}$ ) was mixed with an equal proportion of unlabeled plasmid and complexed with Her-NLS protein followed by further condensation with protamine sulfate. The cells were then incubated with the above complexes for three hours at $37^{\circ} \mathrm{C}$ and $5 \% \mathrm{CO}_{2}$. Subsequently, these were examined by confocal microscopy to monitor the localization of the complexes within cells, along with a counter nuclear Hoechst 33258 stain. The image acquisition conditions for all the experiments were identical.

\section{Preparation of transfection complexes}

Plasmid DNA pCMV $\beta$-gal or $\mathrm{pEGFPN}_{3}$ was purified using the Qiagen kit (endotoxin-free; Qiagen, GmbH, Hilden, Germany) according to the manufacturer's protocol. Complexes were prepared by taking $0.9 \mu \mathrm{g}$ of plasmid DNA and varying amounts of the fusion protein to obtain the desired charge ratio and incubated for 25 minutes at room temperature in Hepes-buffered saline ( $\mathrm{pH}$ 7.4). Transfection experiments were initially performed with Her-NLS and DNA complexes at varying charge ratios as described earlier. A charge ratio of 1:8 was found to be optimal for transfection. Protamine sulfate was added to these complexes to condense the DNA further at a charge ratio of DNA:protein:protamine sulfate 1:8:2 and incubated for 25 minutes at room temperature. Complexes were then diluted with serum-free DMEM to a final volume of $300 \mu \mathrm{L}$. This was divided evenly into $100 \mu \mathrm{L}$ volumes on a 96-well plate format containing 20,000 cells per well, to generate a triplicate set. The cells were incubated for at least three hours, following which complete medium with serum was added, and the reporter gene expression was assayed for $\beta$-galactosidase 24-48 hours post-transfection and the data analyzed as previously described. ${ }^{17}$

\section{MTT assay}

MDA-MB-231 and MDA-MB-453 were grown in a 96-well format to about $70 \%$ confluency. Complexes were prepared with DNA and Her-NLS and evaluated for their toxicity to cells by performing the MTT assay. Cells were incubated with transfection complexes for three hours at $37^{\circ} \mathrm{C}$ in serum-free DMEM. Soon after transfection, cells were rinsed with phosphate-buffered saline and replaced with $75 \mu \mathrm{L}$ DMEM and $25 \mu \mathrm{L}$ MTT ( $2 \mathrm{mg} / \mathrm{mL}$ in phosphate-buffered saline) for three hours, after which the medium was aspirated and replaced with $100 \mu \mathrm{L}$ dimethyl sulfoxide:methanol 1:1 (v/v) and mixed to dissolve the formazan crystals. Absorbance was then measured at $540 \mathrm{~nm}$, with untreated cells serving as controls. ${ }^{17}$

\section{Results}

\section{Generation of plasmid pHer-NLS, overexpression, and purification of Her-NLS chimera}

The motivation for this study originated from a need to add specificity to transfection peptides, such as the NLS, in order to target DNA eventually in a cell-specific manner into the nucleus. In this study, we have generated a recombinant fusion protein designated as Her-NLS, wherein the heregulin ligand was amplified and cloned in frame with three NLS peptides in tandem. Plasmid pHer-NLS, generated through this strategy, encodes an N-terminal His-tag protein which is driven by the $\mathrm{T} 7$ promoter. The reiteration of the NLS epitopes in a cationic fusion peptide, TAT-NLS-Mu, was reported to increase the efficiency of plasmid transfection significantly, as demonstrated earlier ${ }^{4}$ in several mammalian cell lines.

Heregulin- $\alpha$, a natural growth factor ligand, binds with high affinity to heterodimers of HER2/3 or HER2/4 tyrosine kinase receptor, $\mathrm{p} 185^{\mathrm{erbB} 2}$, overexpressed in breast cancer cells, such as MDA-MB-453 and SK-OV-3. The cloned heregulin- $\alpha$ epitope in Her-NLS is the targeting ligand, possessing the epidermal growth factor-like domain. Figure 1A shows the schematic of the fusion construct generated wherein the NLS 
epitopes have been cloned in-frame with the heregulin- $\alpha$ moiety, primarily to facilitate DNA binding and cell-specific targeting. The cloned gene was verified for its authenticity by DNA sequencing prior to growth and overexpression in E. coli. Figure 1B depicts the translated single letter amino acid sequence of Her-NLS. The NLS epitopes and the targeting ligand are color-coded.

\section{Production of Her-NLS by bacterial fermentation}

Plasmid pHer-NLS was transformed and overexpressed in $E$. coli BL21 (DE3) pLysS as an N-terminal His-tag fusion protein. Her-NLS fusion protein was purified by native conditions and characterized for its biochemical and biological properties in vitro. As seen in Figure 1A, the histidine tag at the N-terminus of Her-NLS facilitated

\section{A}

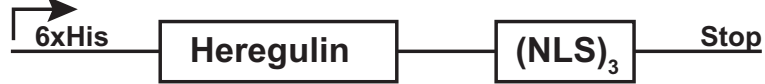

B MRGSHHHHHH GMASMTGGQQ MGRDLYDDDD KDRWGSELLP PQLKEMKSQE SAAGSKLVLR CETSSEYSSL RFKWFKNGNE LNRKNKPQNI KIQKKPGKSE LRINKASLAD SGEYMCKVIS KLGNDSASAN IAIVESNEII TGMPASTEGA YVSSESPIRI SVSTEGANTS SSTSTSTTGT ELYGGSGGSG STMSGYPYDV PDYAGSMAGT DPKKKRKVDP KKKRKVDPKK KRKVGNSKLD PAANKARKEA ELAAATAEQ Stop

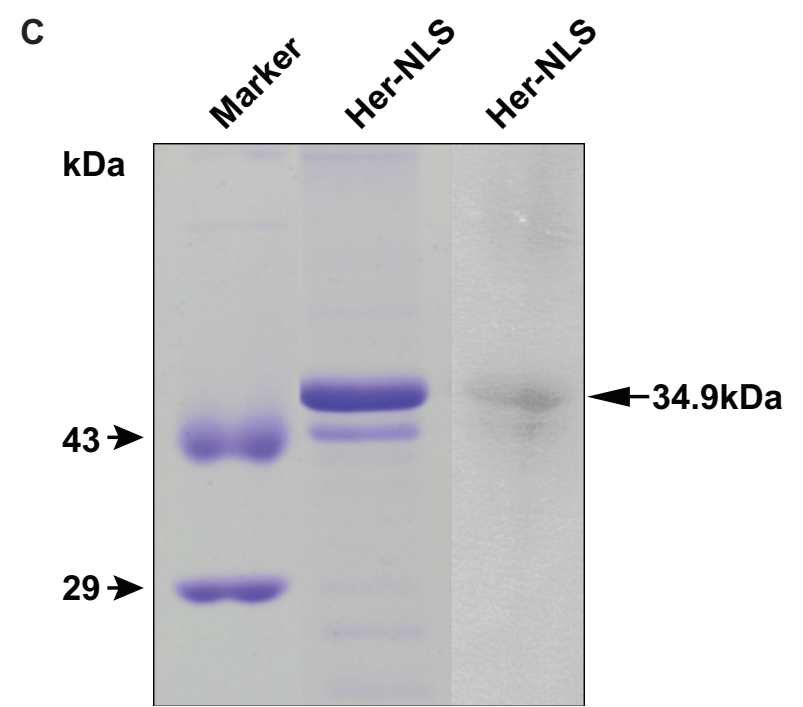

Figure I Construction and gel analysis of DNA-binding carrier protein Her-NLS. A) Schematic representation of chimeric fusion construct encoding the Her-NLS gene. The motifs harboring the NLS sequence (DPKKKRKV) ${ }_{3}$ were cloned in-frame with heregulin and expressed as $\mathrm{N}$-terminal His-tag fusion protein in Escherichia coli. The plasmid is designated as pHer-NLS (not drawn to scale). B) Primary amino acid sequence (single amino acid code is color-coded) of the fusion protein Her-NLS based on DNA sequencing. NLS (blue), heregulin (red), and enterokinase cleavage site (green). The $\mathrm{N}$-terminal His-tag is also depicted (cyan). The predicted molecular weight based on the sequence given is 34,699 Daltons. C) Sodium dodecyl sulfate-polyacrylamide gel electrophoretic analysis of the purified fusion proteins. Lanes correspond to Her-NLS after desalting from PD-10 columns. The molecular weight of the expressed proteins is 34,699 Daltons. Lane on the extreme right shows the immunoblot analysis of HerNLS after electrophoresis and transfer. The $6 \times$ His tagged proteins were detected with monoclonal anti-His antibody followed by secondary antibody goat antimouse IgG alkaline phosphatase conjugate. Detection was by nitro blue tetrazolium chloride and 5-bromo-4-chloro-3-indoyl-I-phosphate treatment.

Abbreviation: NLS, nuclear localization sequence. purification of the recombinant proteins with a cobalt affinity matrix. Fractions were analyzed on $12 \%$ SDS gels, pooled, and desalted with PD-10 columns. Figure 1C shows an SDS-PAGE analysis of the purified protein. This was confirmed by immunoblotting using anti-His antibodies that confirmed the presence of a histidine tag in Her-NLS. The corresponding molecular mass of the protein was further analyzed by MALDI-TOFMS which is 34,654 Daltons (not shown) and close to the expected value of 34,699 Daltons.

\section{DNA binding activity of Her-NLS: protection by condenser protamine sulfate}

Binding being the prerequisite for transfection, we next investigated the ability of purified recombinant Her-NLS to bind plasmid DNA. Complexes of DNA and protein were prepared at charge ratios of 1:8:2 and 1:8:3, incubated at room temperature for 25 minutes, and electrophoresed on an agarose gel. Complexes were viewed after staining with ethidium bromide postelectrophoresis, under ultraviolet light. The ability of Her-NLS protein to bind and protect DNA in the complex was investigated by incubating the complexes with DNase I (Figure 2). DNA binding is evident at charge ratios of $1: 8$. Further protection was observed in the presence of protamine sulfate, lanes 6 and 8. DNase I-treated samples were further extracted with phenol-chloroform and loaded,

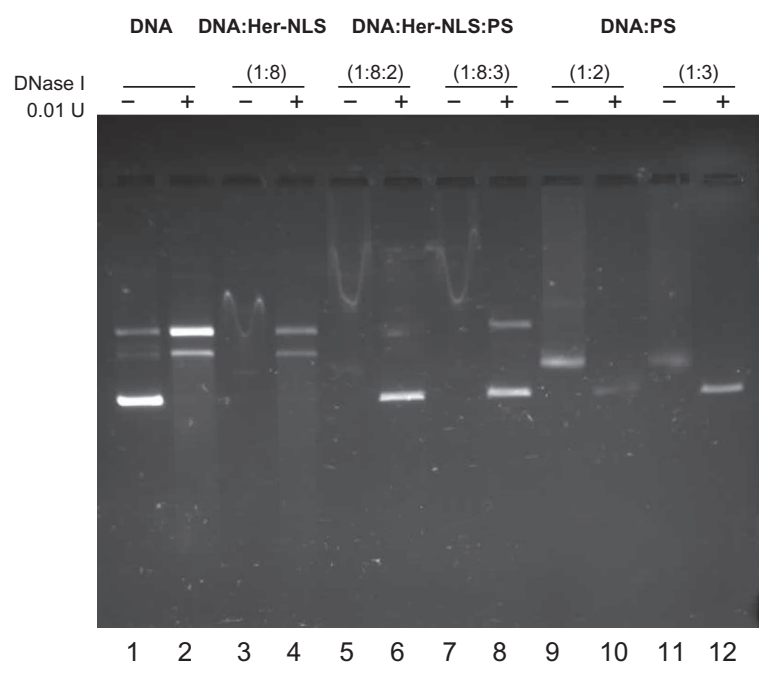

Figure 2 DNase I protection assay of the fusion protein Her-NLS. Plasmid pCMV$\beta$ gal $(0.2 \mu \mathrm{g})$ was incubated in $\mathrm{I} \times$ Hepes buffer saline $(\mathrm{pH} 7.4)$ with increasing amounts of the purified protein corresponding to the charge ratio indicated above lanes 3, 5, and 7 (minus DNase I) and 4, 6, and 8 (plus DNase I). The samples were loaded along with control plasmid DNA (lane I) and in the presence of DNase I (lane 2). Lanes 9 and II indicate untreated DNA:protamine sulfate complexes, while lanes 10 and 12 correspond to DNA:protamine sulfate complexes treated with DNase I. DNase I-treated samples were extracted with phenol-chloroform and loaded along with untreated samples and electrophoresed on I\% agarose gel in Trisacetate-ethylenediaminetetraacetate buffer. Gels were visualized by staining with ethidium bromide.

Abbreviation: NLS, nuclear localization sequence. 
along with untreated samples, and electrophoresed on the same gel. Protected DNA, after phenol extraction, migrated normally as either relaxed or supercoiled DNA in lanes 6, 8, 10 , and 12 , which indicates the extent of protection. Retardation of DNA in lanes 3, 5, and 7 arises from complexes of DNA and Her-NLS at charge ratios of $1: 8,1: 8: 2$, and 1:8:3, respectively.

Our data suggest that Her-NLS binds DNA efficiently, as seen from the retardation of complexes (lanes 3,5 , and 7 ) on $1 \%$ agarose gel. Her-NLS also protects plasmid DNA pEGFPN $_{3}$ from degradation by DNase I (lanes 4, 6, and 8) in the presence of protamine sulfate. The addition of protamine sulfate to DNA:Her-NLS complexes (lanes 5 and 7) has further retarded the mobility of DNA, when compared with the migration of plasmid in lane 3 , indicating enhanced protection in the presence of the condensing agent. As seen in lanes 6 and 8, the complexes are protected from digestion by DNase I, as seen from the intact supercoiled plasmid band. A positive control protamine sulfate complexed with DNA (lanes 9-12), although less retarded on the agarose gel, resulted in the formation of a complex of lesser intensity, indicating the inaccessibility of DNA to ethidium bromide. In the absence of protein in lane 1, which is the control, both relaxed and supercoiled DNA migrated normally.

\section{Her-NLS mediated uptake of DNA into MDA- MB-453 cells in vitro}

Live uptake of chimeric fusion complexes was examined by the addition of DNA:Her-NLS:protamine sulfate complexes at 1:8:2 charge ratio and visualized by confocal microscopy. It is evident that cell-associated particulate fluorescence was higher in MDA-MB-453 compared with MDA-MB-231 cells. Cells counterstained with Hoechst 33258 indicate sections in the nuclear region (Figure 3 ). Western blot analysis shows elevated levels of heregulin receptor expression when compared with MDA-MB-231. As seen from Figure 4, MDAMB-453 cells overexpress the HER2 receptors (185 $\mathrm{kDa})$ when compared with control MDA-MB-231 cells. Chinese hamster ovary cells served as a negative control.

\section{Transfection mediated by Her-NLS in MDA-MB-453}

Having characterized the potential for binding and selective uptake into MDA-MB-453, we performed transient transfection assays in vitro with the pCMVb-gal DNA:Her-NLS:PS complexes at a ratio of $1: 8: 2$. This was to demonstrate primarily the targetability for HER2 receptors in MDAMB-453 (high HER2 expression) and MDA-MB-231 (low HER2 expression). MDA-MB-231 cells with low HER2 levels was used as a control. This was on the premise that the targeting ligand would be recognized by HER2/3 receptors
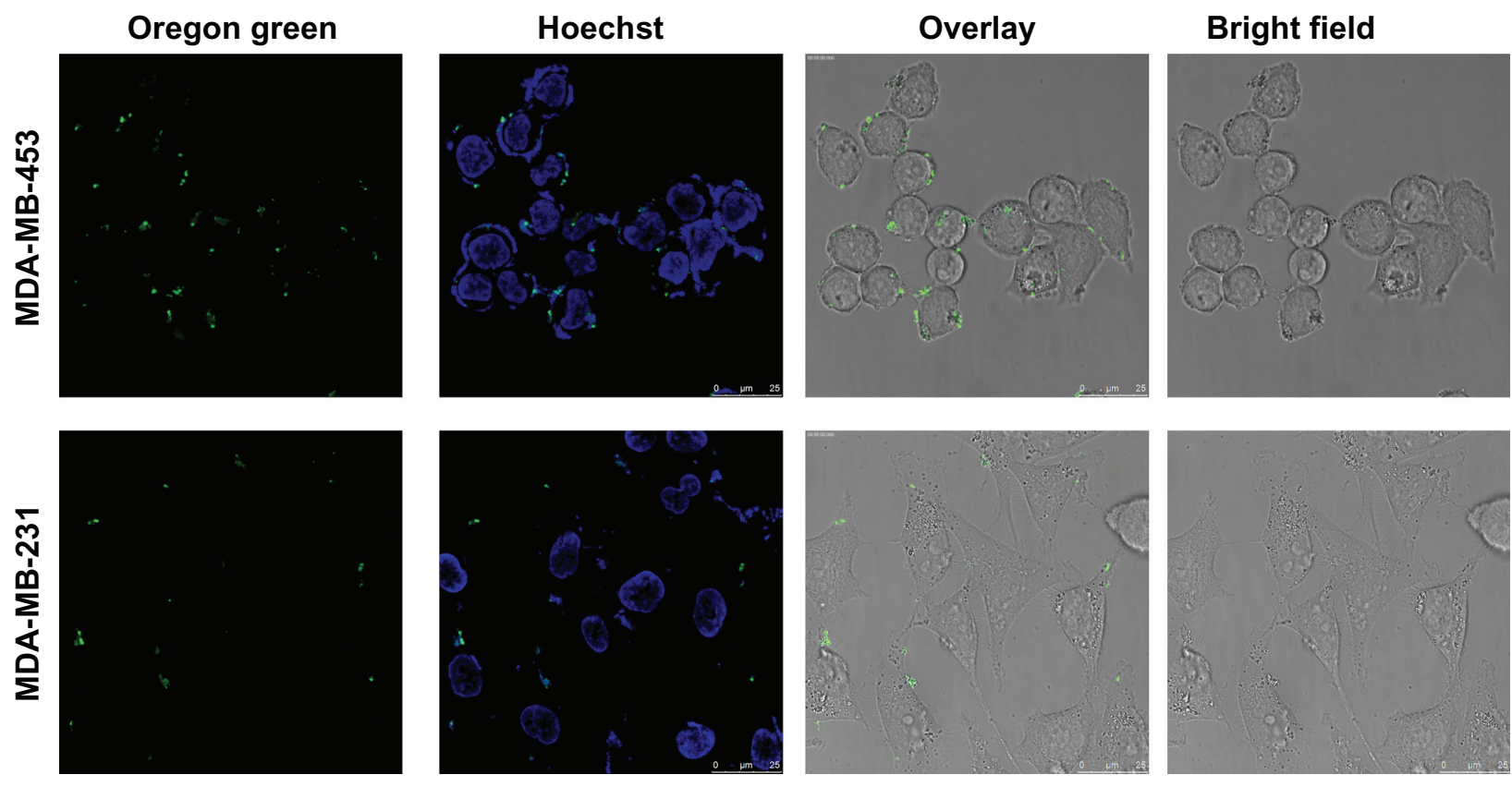

Figure 3 Confocal microscopy of uptake of transfection-competent complexes into MDA-MB-453 versus MDA-MB-23I cells. Confocal microscope analysis of uptake of Oregon green labeled-DNA:Her-NLS fusion protein complexes into cells. Peptide-mediated uptake of labeled DNA into MDA-MB-453 and MDA-MB-23I is seen as cell-associated fluorescence. DNA $0.9 \mu \mathrm{g}$ was used to make the transfection complexes at a charge ratio of I:8:2 (DNA:peptide:protamine sulfate) and applied to cells in the absence of serum. Uptake of the complexes is for three hours' duration, after which the cells were counterstained with Hoechst 33258 , and visualized by confocal microscopy as described.

Abbreviation: NLS, nuclear localization sequence. 

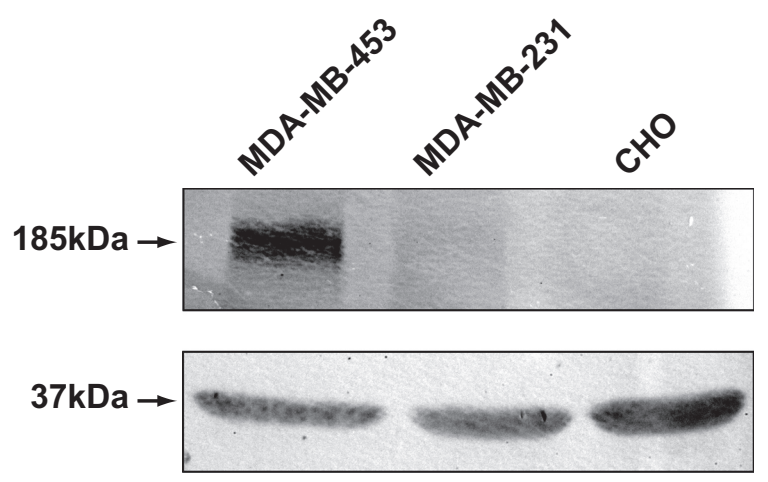

Figure 4 Expression of HER2/3 receptors in MDA-MB-23I and MDA-MB-453 cells by Western blotting. MDA-MB-453 and MDA-MB-23I cell lysates, normalized for protein, were analyzed on $6 \%$ sodium dodecyl sulfate-polyacrylamide gel electrophoretic analysis and blotted onto a nitrocellulose membrane. The blot was probed using primary antibody ErbB2 (intracellular domain) against HER2/3 receptors and treated with the secondary antibody antimouse $\lg G$ conjugated to alkaline phosphatase. Chinese hamster ovary cells were loaded as additional controls. Lysates for probing with antibodies to glyceraldehyde-3-phosphate dehydrogenase was also normalized and loaded separately on a $12 \%$ gel, and served as an internal loading control.

overexpressed in MDA-MB-453 cells. The addition of protamine sulfate to transfection complexes, as seen from the DNA-binding assays, offered further protection to DNA from degradation by nucleases, as seen from the enhanced reporter gene expression, $\beta$-galactosidase, mediated by $\mathrm{D}$ :Her-NLS:protamine sulfate, that is approximately seven-fold higher than D:Her-NLS in MDA-MB-453 cells (see Figure 5). Cationic entities such as protamine, ${ }^{13,18}$ polylysine, or NLS-containing peptides ${ }^{19,4}$ have been widely evaluated for their gene delivery potential and are frequently used to condense and protect plasmid DNA. As seen from

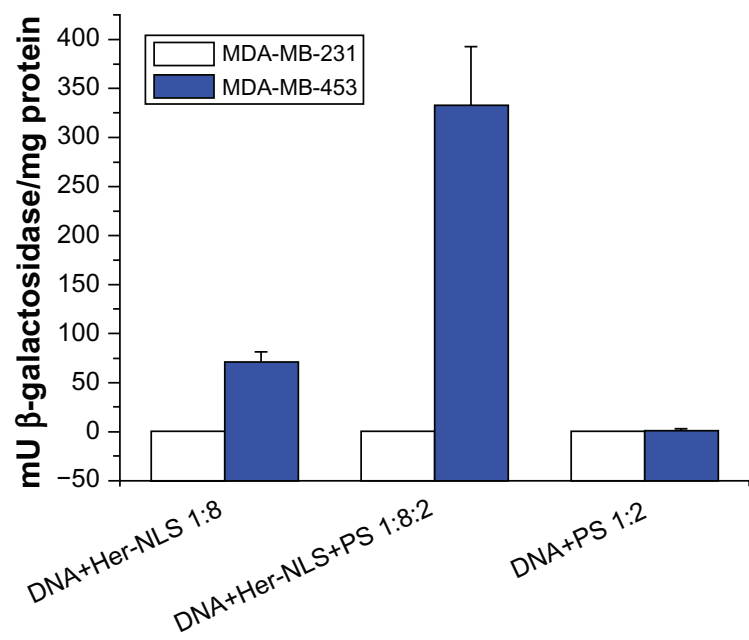

Figure 5 Transient transfection of MDA-MB-453 cells versus MDA-MB-23I cells with Her-NLS fusion protein. Complexes were prepared with the fusion protein at a charge ratio of $\mathrm{I}: 8$ and $1: 8: 2$ of DNA+Her-NLS and DNA+Her-NLS+PS respectively. $\beta$-galactosidase activity was determined 48 hours post transfection. The plot depicts normalized $\mathrm{mU} \beta$-galactosidase activity from plasmid pCMV $\beta$-gal.

Abbreviations: NLS, nuclear localization sequence; PS, protamine sulphate. the graph, under these conditions, a control cell line MDAMB-231, expressing basal levels of HER2/3, was not transfected. Cationic peptide TNM or the Mu peptide that was used as a condenser, like protamine sulfate, conferred protection of DNA from degradation and brought about similar enhancement in reporter gene expression (data not shown).

To examine if Her-NLS related toxicity had any effect on transfection efficiency, we also evaluated the effect of DNA:Her-NLS complexes on MDA-MB-231 and MDAMB-453 cells through an MTT assay (Figure 6) that indicated no apparent toxicity. This implies that these complexes are nontoxic to both MDA-MB-453 and MDA-MB-231 cells at the charge ratios tested. The fact that only MDAMB-453 cells were transfected indicates that the complexes were internalized by receptor-mediated endocytosis. It is purported that the cationic NLS epitopes may serve a dual role in terms of binding DNA and also nuclear localization, while the heregulin epitope recognizes the target receptor in MDA-MB-453 cells. NLS peptides alone do not have the specificity that is observed with Her-NLS (unpublished observations). Our data suggests that through modular design it is possible to achieve cell-specific targeting as a means of facilitating plasmid DNA delivery through receptor-ligand interactions. Such a strategy would enable the replacement of the heregulin ligand with other targeting moieties that would be suited to cell-specific delivery.

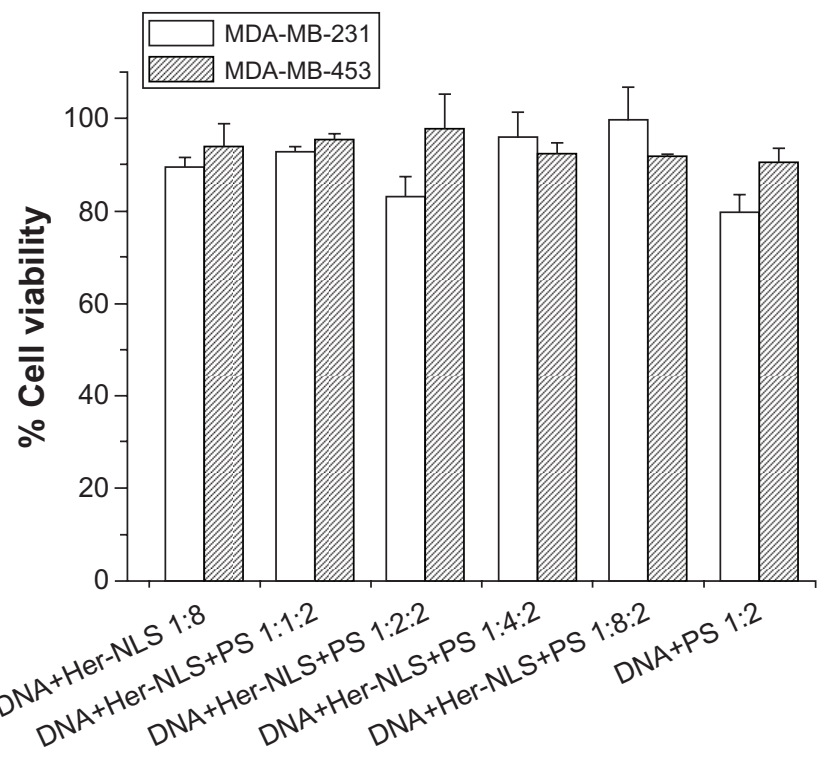

Figure 6 Toxicity assay. The cytotoxicity of DNA-Her-NLS:protamine sulfate complexes at varying concentrations of Her-NLS was evaluated in MDA-MB-23I and MDA-MB-453 cells. The concentration of DNA:protamine sulfate was kept constant at 1:2 charge ratios. Toxicity was measured as percent viability from absorption values obtained using reduced formazan, with cells in the absence of the complexes taken to be 100 .

Abbreviations: NLS, nuclear localization sequence; PS, protamine sulphate. 


\section{Discussion}

Peptides with targeting ligands are widely used in formulations that facilitate specific DNA delivery into cells. Prior to the initiation of this work, it was hypothesized that fusion of the heregulin ligand with NLS would permit DNA binding, followed by uptake as a consequence of receptor-mediated endocytosis. In this study, we have successfully developed a novel, NLS-based peptide delivery system that can target ErbB2-overexpressing MDA-MB-453 breast cancer cells in vitro. We have demonstrated that Her-NLS indeed binds DNA and forms complexes, as seen from the particulate fluorescence in transfected MDA-MB-453 cells. Reporter gene expression was observed only in MDA-MB-453 cells, indicating that Her-NLS targets MDA-MB-453 breast cancer cells specifically at a ratio of 1:8:2 DNA:Her-NLS:protamine sulfate. It is evident that condensers, such as protamine sulfate used in the transfection assays, prevented the degradation of DNA by serum proteins because poor efficiencies were observed in its absence. We observed no increase in transfection efficiency with Her-NLS/protamine sulfate alone on MDA-MB-453 cells. MDA-MB-231 with low levels of heregulin receptors is not transfected, indicating that receptorligand interactions are crucial. As a consequence of these interactions, plasmid DNA gains entry specifically into MDAMB-453 that harbors an excess of HER $2 / 3$ receptors.

The approach of attaching protein-based targeting ligands for cell-specific targeting has been demonstrated earlier by Medina-Kauwe et al. ${ }^{20}$ In this study, the authors fused the penton base to polylysine and heregulin that enables targeting breast cancer cells specifically, while retaining the property of DNA binding through a polylysine stretch of 10 residues and also facilitating endosomal escape. A linear sequence of positive charges, as in poly-L-lysine, has earlier been shown to be insufficient for transfection, ${ }^{21}$ indicating the role of peptide sequences for efficient binding and DNA condensation. Although polylysine has been used extensively in gene delivery, ${ }^{22,23}$ it is accompanied with considerable toxicity, ${ }^{24,25}$ and is now used only when chemically modified.

A major limitation to gene transfer is the entry of plasmid DNA from the cytoplasm to the nucleus. Strategies for the use of NLS bioconjugates that utilize the cytoplasmic machinery to shuttle bound nucleic acids efficiently into the nucleus have been discussed. ${ }^{26}$ Xavier et al have demonstrated that a recombinant peptide with three NLS epitopes in tandem, facilitates DNA delivery. ${ }^{4}$ The Her-NLS generated in this study apparently increases nuclear entry soon after receptor-mediated internalization of the complexes in MDA-MB-453 cells. It is possible that the binding efficiency of Her-NLS to DNA is facilitated by the presence of arginine and lysine residues present in multiple copies or overall basicity of the protein. The NLS epitopes may enable rapid transit of endocytosed complexes which are then rapidly delivered into the nucleus, possibly by interaction with importins in the cellular milieu, resulting in enhancement of $\beta$-galactosidase reporter gene expression in MDA-MB-453 cells. Puri et al have observed that HER2 specific affibody-conjugated liposomes improve the delivery of anticancer agents in a cell-specific manner. ${ }^{8}$ These efforts require the use of formulations that would be suitable for combination therapies. Our strategy employs two epitopes, engineered to bind DNA and target cancer cells overexpressing the heregulin receptor in vitro. At the optimal ratios, complexes are internalized in MDA-MB-453 human breast cancer cells, possibly via receptor-specific binding and internalization into the cytoplasm followed by rapid entry into the nucleus. Her-NLS renders efficient targeting in vitro via the cloned heregulin. Furthermore, the NLS peptides fused to the Her moiety, through its NLS (PKKKRK) ${ }_{3}$-enhanced nuclear entry, resulting in high gene expression in vitro. This delivery system lays the foundation for further development of effective therapeutic molecules for delivery of plasmid DNA and other siRNA. The study also demonstrates the scope for further development of nonviral carriers by generating minimal sequences for cell-specific nucleic acid delivery. This is possibly the first demonstration with NLS-containing epitopes fused to heregulin ligand for efficient targeting into cells expressing the HER2 receptor. Apart from TAT, other cationic-nucleic acid-binding modules, such as the NLS or multiple copies of $\mathrm{Mu}$, could be cloned along with doublestranded RNA-binding domain and targeting ligands in one frame to introduce multifunctionality. This would permit specific interaction with the siRNA that can target cells specifically via epitopes engineered into one recombinant protein molecule. This also translates to a multidomain fusion peptide that would be a single entity having modules that could be replaced depending on the recipient cell. It is also possible to replace heregulin, the targeting ligand, with other peptide ligands that recognize its corresponding receptors on other cell types in the context of siRNA or plasmid DNA delivery. This work is currently in progress.

\section{Acknowledgments}

The authors wish to acknowledge support from the Department of Science and Technology, India, through research grant SR/ SO/BB-046/2007. Partial support from a CSIR network project \# MWP0035 is acknowledged. VG specially acknowledges Dr Medina-Kauwe for providing the cell lines and plas- 
mid constructs used in this study. We acknowledge Bhanu Ramanand for determining the molecular mass, Nandini Rangarajan for help with confocal microscopy, and Narayan Nagesh for sequencing the plasmid clones. We gratefully acknowledge Srinath Govindarajan for help with the revision experiment. We also acknowledge A Kalyani, our summer intern, for generating the recombinant NLS construct.

\section{Disclosure}

The authors report no conflicts of interest in this work.

\section{References}

1. Abes S, Moulton H, Turner J, et al. Peptide-based delivery of nucleic acids: Design, mechanism of uptake and applications to splice-correcting oligonucleotides. Biochem Soc Trans. 2007;35:53-55.

2. Lundberg P, El-Andaloussi S, Sutlu T, et al. Delivery of short interfering RNA using endosomolytic cell-penetrating peptides. FASEB $J$. 2007;21:2664-2671.

3. Vives E. Present and future of cell-penetrating peptide mediated delivery systems: "Is the Trojan horse too wild to go only to Troy?" J Control Release. 2005;109:77-85.

4. Xavier J, Singh S, Dean DA, et al. Designed multi-domain protein as a carrier of nucleic acids into cells. $J$ Control Release. 2009;133: $154-160$.

5. Rajagopalan R, Xavier J, Rangaraj N, et al. Recombinant fusion proteins TAT-Mu, $\mathrm{Mu}$ and $\mathrm{Mu}-\mathrm{Mu}$ mediate efficient non-viral gene delivery. J Gene Med. 2007;9:275-286.

6. Fominaya J, Wels W. Target cell-specific DNA transfer mediated by a chimeric multidomain protein. Novel non-viral gene delivery system. J Biol Chem. 1996;27:10560-10568.

7. Faltus T, Yuan J, Zimmer B, et al. Silencing of the HER2/neu gene by siRNA inhibits proliferation and induces apoptosis in HER2/neuoverexpressing breast cancer cells. Neoplasia. 2004;6:786-795.

8. Puri A, Kramer-Marek G, Campbell-Massa R, et al. HER2-specific affibody-conjugated thermosensitive liposomes (affisomes) for improved delivery of anticancer agents. J Liposome Res. 2008;18:293-307.

9. Tan M, Keng-Hsueh L, Yao J, et al. Selective inhibition of ErbB2-overexpressing breast cancer in vivo by novel TAT-based ErbB2-targeting signal transducers and activators of transcription 3-blocking peptide. Cancer Res. 2006;66:3764-3772.

10. Bidwell GL III, Davis AN, Raucher D. Targeting a c-Myc inhibitory polypeptide to specific intracellular compartments using cell penetrating peptides. J Control Release. 2009;135:2-10.
11. Holmes WE, Sliwkowski MX, Akita RW, et al. Identification of heregulin, a specific activator of p185erbB2. Science. 1992;256: 1205-1210.

12. Kern JA, Schwartz DA, Nordberg JE, et al. p185neu expression in human lung adenocarcinomas predicts shortened survival. Cancer Res. 1990;50:5184-5187.

13. Medina-Kauwe LK, Maguire M, Kasahara N, et al. Nonviral gene delivery to human breast cancer cells by targeted Ad5 penton proteins. Gene Ther. 2001;8:1753-1761.

14. Canine BF, Wang Y, Hatefi A. Biosynthesis and characterization of a novel genetically engineered polymer for targeted gene transfer to cancer cells. J Control Release. 2009;138:188-196.

15. ArthanariY, Pluen A, Rajendran R, et al. Delivery of therapeutic shRNA and siRNA by Tat fusion peptide targeting bcr-abl fusion gene in chronic myeloid leukemia cells. J Control Release. 2010;145:272-280.

16. Subramanian A, Ranganathan P, Diamond SL. Nuclear targeting peptide scaffolds for lipofection of nondividing mammalian cells. Nat Biotechnol. 1999; 17:873-877.

17. Gopal V, Prasad TK, Rao NM, et al. Synthesis and in vitro evaluation of glutamide-containing cationic lipids for gene delivery. Bioconjug Chem. 2006;17.6:1530-1536.

18. Zhang JS, Li S, Huang L. Cationic liposome-protamine-DNA complexes for gene delivery. Methods Enzymol. 2003;373:332-342.

19. Collins E, Birchall JC, Williams JM, Gumbleton M. Nuclear localisation and pDNA condensation in non-viral gene delivery. J Gene Med. 2007;9:265-274.

20. Medina-Kauwe LK, Kasahara N, Kedes L. 3PO, a novel nonviral gene delivery system using engineered Ad5 penton proteins. Gene Ther. 2001;8:795-803.

21. Hyndman L, Lemoine JL, Huang L, Porteous JD, Boyd AC, Nan X. HIV-1 TAT protein transduction domain peptide facilitates gene transfer in combination with cationic liposomes. J Control Release. 2004; 99:435-444.

22. Wu GY, Wu CH. Evidence for targeted gene delivery to Hep G2 hepatoma cells in vitro. Biochemistry. 1988;27:887-892.

23. Yu H, Chen X, Lu T, et al. Poly(L-lysine)-graft-chitosan copolymers: Synthesis, characterization, and gene transfection effect. Biomacromolecules. 2007;8:1425-1435.

24. Hill IR, Garnett MC, Bignotti F, Davis SS. In vitro cytotoxicity of poly(amidoamine)s: Relevance to DNA delivery. Biochim Biophys Acta. 1999;1427:161-174.

25. Szabo R, Mezo G, Pallinger E, et al. In vitro cytotoxicity, chemotactic effect, and cellular uptake of branched polypeptides with poly[L-lys] backbone by $\mathrm{J} 774$ murine macrophage cell line. Bioconjug Chem. 2008; 19:1078-1086.

26. Escriou V, Carriere M, Scherman D, et al. NLS bioconjugates for targeting therapeutic genes to the nucleus. Adv Drug Deliv Rev. 2003;55:295-306.
International Journal of Nanomedicine

\section{Publish your work in this journal}

The International Journal of Nanomedicine is an international, peerreviewed journal focusing on the application of nanotechnology in diagnostics, therapeutics, and drug delivery systems throughout the biomedical field. This journal is indexed on PubMed Central, MedLine, CAS, SciSearch ${ }^{\circledR}$, Current Contents ${ }^{\circledR} /$ Clinical Medicine,

\section{Dovepress}

Journal Citation Reports/Science Edition, EMBase, Scopus and the Elsevier Bibliographic databases. The manuscript management system is completely online and includes a very quick and fair peer-review system, which is all easy to use. Visit http://www.dovepress.com/ testimonials.php to read real quotes from published authors. 